\title{
Molecular alterations as target for therapy in metastatic osteosarcoma: a review of literature
}

\author{
J. PosthumaDeBoer $\cdot$ M. A. Witlox $\cdot$ \\ G. J. L. Kaspers · B. J. van Royen
}

Received: 15 July 2010/ Accepted: 18 March 2011/Published online: 2 April 2011

(C) The Author(s) 2011. This article is published with open access at Springerlink.com

\begin{abstract}
Treating metastatic osteosarcoma (OS) remains a challenge in oncology. Current treatment strategies target the primary tumour rather than metastases and have a limited efficacy in the treatment of metastatic disease. Metastatic cells have specific features that render them less sensitive to therapy and targeting these features might enhance the efficacy of current treatment. A detailed study of the biological characteristics and behaviour of metastatic OS cells may provide a rational basis for innovative treatment strategies. The aim of this review is to give an overview of the biological changes in metastatic OS cells and the preclinical and clinical efforts targeting the different steps in OS metastases and how these contribute to designing a metastasis directed treatment for OS.
\end{abstract}

J. PosthumaDeBoer · B. J. van Royen $(\varangle)$

Department of Orthopaedic Surgery, VU University Medical

Center, De Boelelaan 1117, 1081 HV Amsterdam, The

Netherlands

e-mail: bj.vanroyen@vumc.nl

\section{A. Witlox}

Department of Orthopaedic Surgery, Westfries Gasthuis,

Hoorn, The Netherlands

\section{G. J. L. Kaspers}

Paediatric Oncology/Haematology, VU University Medical

Center, Amsterdam, The Netherlands

B. J. van Royen

VU University Medical Center, PO Box 7057,

1007 MB Amsterdam, The Netherlands
Keywords Drug resistance - Metastasis .

Osteosarcoma · Therapy

$\begin{array}{ll}\text { Abbreviations } \\ \text { Bcl-2 } & \text { B cell lymphoma 2 associated oncogene } \\ \text { Bcl-XL } & \text { Bcl2-like 1 } \\ \text { CXCR4 } & \text { Chemokine (C-X-C-motif) receptor } 4 \\ \text { CXCL } & \text { Chemokine (C-X-C-motif) ligand } \\ \text { ECM } & \text { Extracellular matrix } \\ \text { EGFR } & \text { Epidermal growth factor receptor } \\ \text { ERK } & \text { Extracellular signal regulated kinase } \\ \text { FAK } & \text { Focal adhesion kinase } \\ \text { GH } & \text { Growth hormone } \\ \text { HLA } & \text { Human leukocyte antigen } \\ \text { IGF-1R } & \text { Insulin-like growth factor 1 receptor } \\ \text { IFN- } \alpha & \text { Interferon-alpha } \\ \text { IL } & \text { Interleukin } \\ \text { JAK } & \text { Janus kinase } \\ \text { mAB } & \text { Monoclonal antibody } \\ \text { MAP(K) } & \text { Mitogen-activated protein (kinase) } \\ \text { MMP } & \text { Matrix metalloproteinase } \\ \text { MTP-PE } & \text { Muramyl tripeptide phosphatidyl ethanolamine } \\ \text { mTOR } & \text { Mammalian target of rapamycin } \\ \text { NF- } \kappa \text { B } & \text { Nuclear factor-kappa B } \\ \text { NK cells } & \text { Natural killer cells } \\ \text { PI3K } & \text { Phosphatidylinositol 3-kinase } \\ \text { PDGF-R } & \text { Platelet-derived growht factor receptor } \\ \text { OS } & \text { Osteosarcoma } \\ \text { SARC } & \text { Sarcoma Alliance for Research through } \\ & \text { Collaboration } \\ \text { STAT } & \text { Signal transducer and activator of } \\ & \text { transcription } \\ \text { TGF- } \beta & \text { Transforming growth factor-beta } \\ \text { VEGF } & \text { Vascular endothelial growth factor } \\ \text { WIF-1 } & \text { Wnt inhibitory factor 1 } \\ & \end{array}$




\section{Introduction}

Osteosarcoma (OS) is the most common primary malignant bone tumour in children and adolescents. The estimated incidence rate worldwide is $4 /$ million/year, with a peak incidence at the age of 15-19 years [1]. In OS there is a high tendency to metastatic spread. Approximately $20 \%$ of patients present with lung metastases at initial diagnosis and, additionally, in $40 \%$ of patients metastases occur at a later stage. Eighty percent of all metastases arise in the lungs, most commonly in the periphery of the lungs, and exhibit resistance to conventional chemotherapy [2-7]. The 5 -year survival rate for OS patients with metastases is $20 \%$ compared to $65 \%$ for patients with localised disease and most deaths associated with OS are the result of metastatic disease [5, 8-11].

For patients with pulmonary metastasis, especially those who have metastasis at initial diagnosis, the combination of radical metastasectomy and chemotherapy offers the best outcome and even potential cure. Nevertheless, recurrent development of pulmonary metastases after initial radical metastasectomy is reported to be high and repeated metastasectomies are sometimes performed. As metastasectomy does yield an improved survival in most patients it should therefore always be performed when feasible [2, 4, 12-14].

In order to improve survival, the ultimate questions to be answered are: Why does OS metastasize, particularly to the lungs? And, more importantly: Why does therapy fail in metastatic disease? In this regard, we hypothesise that drug resistance is a key issue in the failure to control metastatic disease. It has been shown that OS lung metastases display a biological behaviour different from the primary tumours [2, 14-27]. Metastases are comprised of cell clones that differ from primary tumours with respect to ploidy, enzyme profile, karyotype and chemosensitivity [2, 28-32]. Therapeutic regimens that target primary tumours are therefore unlikely to be successful in the treatment of metastatic disease.

Metastasis is considered to be the final though most critical step in tumorigenesis of malignant tumours [33]. The metastatic cancer cells subsequently complete the following steps: Invasion through the extracellular host matrix and entrance into the circulation (I), survival in the circulation (II) and evasion of the host immune system (III), arrest and extravasation at a target organ site (IV), adherence and survival in the target organ microenvironment (V, VI) and finally formation of neovasculature to allow growth at the target organ site (VII) [14, 33-36]. Each step is of equal importance and must be fully completed by the tumour cell to achieve successful metastasis. The altered biological behaviour in metastatic cells is the result of specific molecular changes. We will discuss each of these specific steps with special attention to the molecules involved in OS metastasis (Table 1) and implications for therapy. Over the last decade, much research has been performed to try to unravel the biology of OS metastasis and many (pre)clinical studies have attempted to discover new treatment options for metastatic OS. For example, gene expression profiling of metastatic cells using a cDNA microarray approach has identified genes responsible for metastasis [27, 37-39]. Also, expression levels of specific proteins in OS lung metastases have been analysed. In these studies, expression levels of proteins involved in metastases link the molecular aberrancies to clinical outcome in terms of survival rates. These alterations may also provide novel drug targets [21, 23, 25, 36, 40-42]. Table 2 summarises (pre)clinical studies for treating OS metastases.

The aim of this review is to give an overview of the biology of metastatic OS cells and of (pre)clinical efforts targeting the different steps in OS metastases and how these may contribute to designing a metastasis directed treatment for OS.

\section{OS metastasis}

\section{(I) Migration and invasion}

Migration of cells away from the primary tumour and invasion through the extracellular matrix (ECM) towards the bloodstream is considered the first step contributing to metastasis. In OS, it has been described that MetalloProteinases (MMPs) 2 and 9 and m-Calpain play a role in degradation of the ECM $[10,14,16,18,19,27,34,35$, 43-46]. Also, the $\mathrm{Wnt} / \beta$-catenin pathway and Src-kinase are implicated as inducers of migration [9, 35, 46, 47]. In $\mathrm{OS}$, Notch is a relatively recently identified pathway and has been identified as a promoter of invasion in OS. In highly metastatic OS cell lines there is an upregulation of the Notch1 and Notch2 receptor, as well as the Notch induced gene Hes1. In patient samples, expression of the Hes1 gene inversely correlated with survival [41, 48-50].

\section{(Pre)clinical studies: Notch}

In a preclinical setting, downregulation of the Notch signalling pathway has been shown to impair the invasiveness of cell lines, but has no effect on cell proliferation or in vitro tumorigenesis. Notch-inhibited cell lines had less potential to form lung metastases in an orthotopic mouse model when compared to untreated cell lines. The exact mechanism through which inhibition of the Notch pathway and its target gene Hes1 leads to reduced invasion still remains unknown [41, 50]. 
Table 1 Steps of metastasis in OS and molecular alterations that contribute to each process

\begin{tabular}{|c|c|c|c|}
\hline & Steps of metastasis & Molecular involvement & References \\
\hline \multirow[t]{5}{*}{ I } & \multirow[t]{5}{*}{ Migration and invasion } & MMPs & $\begin{array}{l}{[10,14,16,18,19,27,34,35,44-} \\
46]\end{array}$ \\
\hline & & m-Calpain & {$[35,43]$} \\
\hline & & Wnt & {$[9,35,46,47]$} \\
\hline & & Src & {$[35,44]$} \\
\hline & & Notch & {$[41,48-50]$} \\
\hline \multirow[t]{10}{*}{ II } & \multirow[t]{6}{*}{ (a) Anoikis resistance } & PI3K/Akt & {$[9,14,16,51]$} \\
\hline & & $\mathrm{Src} / \mathrm{PI} 3 \mathrm{k} / \mathrm{Akt}$ & {$[9,14,16,44]$} \\
\hline & & Src/Ras/MAPK & {$[18,35]$} \\
\hline & & $\mathrm{NF}-\kappa \mathrm{B}$ & {$[27]$} \\
\hline & & $\mathrm{Wnt} / \beta$-catenin & [14] \\
\hline & & BcL family & {$[9,35,51]$} \\
\hline & \multirow[t]{4}{*}{ (b) Apoptosis resistance } & $\mathrm{Src}$ & {$[9,16,18,35,44]$} \\
\hline & & $\mathrm{NF}-\kappa \mathrm{B}$ & {$[27,44,51,67]$} \\
\hline & & $\mathrm{Wnt} / \beta$-catenin & {$[14,17,19,46,47,53,54]$} \\
\hline & & Fas/FasL & {$[5,23,28,36,55,56]$} \\
\hline \multirow[t]{3}{*}{ III } & \multirow[t]{3}{*}{ Evasion of immune system } & HLA-1 & {$[14,60]$} \\
\hline & & IL-10 & [14] \\
\hline & & Fas & {$[62]$} \\
\hline \multirow[t]{4}{*}{ IV } & \multirow[t]{4}{*}{ Arrest and extravasation } & CXCR4-CXCL12 & {$[9,10,14,15,22,42,69]$} \\
\hline & & CXCR3-CXCL9-11 & {$[10]$} \\
\hline & & CXCR4/MMPs & {$[9,10,15]$} \\
\hline & & CXCR3-4/Erk/NF- $\kappa \mathrm{B}$ & {$[10,67]$} \\
\hline \multirow[t]{3}{*}{ V } & \multirow[t]{3}{*}{ Adherence } & Ezrin/MAPK/Akt & {$[14,21,25,35]$} \\
\hline & & Ezrin/ $\beta 4$-Integrin/PI3K & {$[70]$} \\
\hline & & CD44/Akt/mTOR & {$[14,19,21]$} \\
\hline \multirow[t]{5}{*}{ VI } & \multirow[t]{5}{*}{ Dormancy } & Integrin- $\alpha 5 \beta 1$ & {$[73,74]$} \\
\hline & & Integrin- $\alpha 5 \beta 1 /$ Erk/p38 & {$[14,35]$} \\
\hline & & Bcl-XL & {$[14,35]$} \\
\hline & & IGF/PI3K & {$[75]$} \\
\hline & & ECM & {$[73,74]$} \\
\hline \multirow[t]{8}{*}{ VII } & \multirow[t]{8}{*}{$\begin{array}{l}\text { Angiogenesis and } \\
\text { proliferation }\end{array}$} & $\begin{array}{l}\text { EGFR. PDGFR, VEGF, IGFR, } \\
\text { TGF- } \beta\end{array}$ & {$[9,14,35,40,68,77,78,81]$} \\
\hline & & MMPs & {$[9,44,78]$} \\
\hline & & VEGF/Erk/NF- $\kappa \mathrm{B}$ & {$[35,68]$} \\
\hline & & VEGF/PI3K & {$[35,40]$} \\
\hline & & EGFR/Src/Ras/MAPK/STAT3 & {$[9,18]$} \\
\hline & & Src & {$[14,35,44]$} \\
\hline & & Integrin/PI3K/Erk1-2 & {$[9,35,67,75]$} \\
\hline & & Wnt/ $\beta$-catenin/CyclinD-Survivin & {$[46,52]$} \\
\hline
\end{tabular}

(IIa) Survival in the bloodstream

Dissemination of cancer cells through the body requires the cells to survive in the circulation. Non-malignant cells, as well as non-metastatic tumour cells, become apoptotic after loss of cell-cell adhesions or interaction with the ECM in their original tissue. This specific mode of apoptosis is called anoikis. One reason for this type of apoptosis to occur is that Integrin signalling ceases to exist in solitary cells. Under adherent conditions, survival is often mediated through Integrin signalling pathways in which Focal adhesion kinase (FAK) is a central player. FAK activates the important PI3K/Akt survival pathway. Metastatic tumour cells evade anoikis by intrinsically activated survival pathways via for example PI3K or Akt signalling, independent of extrinsic Integrin and FAK signalling [9, 14, 16, 51]. Src kinase can also activate the PI3K/Akt and the Ras/MAPK survival pathways independent of FAK 
Table 2 Preclinical and clinical studies targeting specific molecules in OS metastasis

\begin{tabular}{|c|c|c|c|c|}
\hline & Steps of metastasis & Target & Drug & References \\
\hline & & Preclinical & & \\
\hline \multirow{9}{*}{$\begin{array}{l}\text { I } \\
\text { II }\end{array}$} & Migration and Invasion & Notch & & {$[41,50]$} \\
\hline & (a) Anoikis resistance & & & \\
\hline & (b) Apoptosis resistance & Preclinical & & \\
\hline & & Wnt & & {$[46,52]$} \\
\hline & & Src & & {$[18]$} \\
\hline & & Clincial & & \\
\hline & & Src & & \\
\hline & & & Dasatinib & {$[60]$} \\
\hline & & & & www.clinicaltrials.gov/NCT00752206 \\
\hline \multirow[t]{9}{*}{ III } & Evasion of & Preclinical & & \\
\hline & & Fas & & \\
\hline & & & IL12 & {$[36,56,62]$} \\
\hline & & & IL18 & {$[63]$} \\
\hline & & & Gemcitabine & {$[55]$} \\
\hline & & Clinical & & \\
\hline & & Fas & & \\
\hline & & & Liposomal MTP-PE & {$[64]$} \\
\hline & & & IFN- $\alpha$ & {$[61,66]$} \\
\hline \multirow[t]{3}{*}{ IV } & Arrest and extravasation & Preclinical & & \\
\hline & & CXCR4 & & [22] \\
\hline & & CXCR3 & & {$[10]$} \\
\hline \multirow[t]{2}{*}{ V } & Adherence & Preclinical & & \\
\hline & & Ezrin & & {$[21,25,45,70]$} \\
\hline VI & Dormancy & & & \\
\hline \multirow[t]{8}{*}{ VII } & Proliferation and & Preclinical & & \\
\hline & & Endostatin & & [83] \\
\hline & & IGF-1R & & {$[79,84]$} \\
\hline & & Clinical & & \\
\hline & & IGF-1R & & \\
\hline & & & OncoLar & [78] \\
\hline & & & R1507 & www.clinicaltrials.gov/NCT00642941 \\
\hline & & & SCH717454 & www.clinicaltrials.gov/NCT00617890 \\
\hline
\end{tabular}

signalling and thus stimulate ainoikis resistance $[9,14,16$, $18,35,44,51]$. Other survival mechanisms are also of importance in the evasion of ainoikis, such as activation the nuclear factor-kappa B $(\mathrm{NF}-\kappa \mathrm{B})$ pathway $[27,51]$ and Wnt-mediated upregulation of the $\beta$-catenin activity. High levels of $\beta$-catenin expression have been shown to be associated with a metastatic phenotype in OS $[14,19,20$, 46]. Finally, overexpression of anti-apoptotic genes such as $\mathrm{Bcl}-2, \mathrm{Bcl}-\mathrm{XL}$ or FAK is exploited by solitary metastatic cells to obtain a survival benefit $[9,35,51]$.

\section{(IIb) Apoptosis resistance}

The survival of tumour cells through all stages of metastasis (not only in the bloodstream) is paramount to successful metastasis. Mechanisms involved in apoptosis resistance throughout metastasis include activation of the Src and NF- $\kappa \mathrm{B}$ pathways and the overexpression of antiapoptotic genes [9, 44, 46, 51, 52]. Wnt-signalling is also involved in resistance to apoptosis throughout other steps of metastasis and is considered to be important for tumour progression in general. Upon binding of Wnt to one of its receptors, $\beta$-catenin degradation in the cytoplasm is prevented. After $\beta$-catenin is stabilised it translocates to the nucleus where it co-regulates oncogene transcription and cell cycle progression and hence promotes survival and proliferation [14, 17, 46, 47, 53, 54].

In established metastases, the tumour cells are confronted with receptor-mediated cell death. Binding of Fas on the surface of metastatic cells to its Fas-ligand (FasL) expressed constitutively on lung tissue, activates the Fasapoptosis pathway and leads to cell death. Cross-linkage of 
Fas with FasL on one cell results in apoptosis as well [36, 55]. Resistance to death-receptor-induced apoptosis is commonly seen and is highly important for the successful maintenance of metastases. The Fas/Fas-ligand pathway is a death receptor pathway that is often down-regulated in metastatic cell populations, rather than in primary tumours $[23,28,36]$. The Fas pathway is also of influence on chemotherapy-induced apoptosis and thus on its therapeutic efficacy [56]. Much (pre) clinical research has been performed concerning this pathway in metastatic OS and promising results have been obtained. These results are discussed following the section on immune evasion, since the Fas pathway plays a role in that as well.

Thus, apoptosis resistance is very much exploited by the metastatic cell and this feature is likely to contribute to resistance to therapy in metastatic OS [51, 52]. The failure to induce apoptosis upon treatment is thought to be the result of a misbalance between pro- and anti-apoptotic signalling. Restoration of this balance, thereby creating an environment in favour of pro-apoptotic signalling could theoretically enhance treatment with cytotoxic agents [9, $35,45,51,56-58]$.

\section{(Pre)clinical studies: Wnt and Src}

The Wnt-pathway is a putative therapeutic target because a majority of OS samples show aberrant activation of this pathway, leading to the transcription of oncogenes and cell cycle progression. This in turn leads to proliferation and enhanced survival $[46,53,59]$. When targeting the Wntpathway, activating mutations in downstream molecules for example $\beta$-catenin can be of negative influence as it may bypass Wnt inhibition and preserve the invasive phenotype of the metastatic cell [19]. A preclinical study by Leow et al. [52] has shown that inhibition of the Wnt/ $\beta$-catenin pathway resulted in lower levels of nuclear $\beta$-catenin, resulting in a decreased expression of $\beta$-catenin target genes. This led to an inhibition of migratory potential through downregulation of MMP-9, and a decrease in expression of Cyclin-D, c-myc and Survivin. The latter was responsible for an antiproliferative effect and an increase in cell death. These results were recently confirmed by Rubin et al. [46], who showed that re-expression of Wnt inhibitory factor 1 (WIF-1), a secreted Wnt-antagonist, inhibited Wnt signalling and reduced tumour growth and metastasis in OS mouse models. These results show a possible therapeutic benefit of Wnt-pathway disruption in the treatment of metastatic OS.

Src-kinase is a kinase that is involved in almost all steps of cancer metastasis, namely in proliferation, adhesion, migration, survival and angiogenesis [44]. Based on its multi-step involvement in metastasis, it could be an interesting therapeutic target in OS metastasis. Pre-clinical work shows that Src inhibition with Dasatinib effectively inhibits Src phosphorylation in primary tumours; however, it did not impair the development of pulmonary metastases. Histopathological analysis of both OS primary tumours and lung nodules showed minimal Src-kinase phosphorylation after treatment with Dasatinib. However, Src-kinase phosphorylation was low in untreated lung metastasis as well. This suggests that Dasatinib was effective in inhibiting Src-pathway activation in OS cells, but it is not clear what the phosphorylation status is during the stages of OS metastasis and how this influences the process [18]. The use of Dasatinib in patients with advanced (osteo)sarcomas was examined recently by the SARC (Sarcoma Alliance for Research through Collaboration) in a phase II clinical trial. Disappointingly, preliminary results show no treatment effect of Dasatinib as a single agent in patients with overt lung metastases [60]. The same group is looking into the effectiveness of Src inhibition with a more specific Srckinase inhibitor (Saracatinib) to obtain progression free survival among patients with resectable OS lung metastases (clinicaltrials.gov/NCT00752206).

\section{(III) Evasion of the immune system}

Another important precondition for the survival of metastatic cells is the evasion of the host immune surveillance throughout all the steps of metastasis. Tumour cells, either circulating or at the site of metastases, can modulate the immune system of the host in order to achieve a survival advantage. Down-regulation of cell surface receptor HLA class 1 is one of such mechanisms. This impairs the recognition of tumour cells by the host cytotoxic T-lymphocytes. Tumour cells can also induce the production of immunosuppressive molecules such as IL-10 [14, 61]. Modulation of the immune system such that it recognizes and destroys (circulating) tumour cells would be a successful anti-metastatic treatment. Interferons are cytokines that can affect the recognition of tumour cells by the immune system by influencing the (re)expression of HLA molecules on the cell surface. Interferons also exert an anti-proliferative effect on OS cells through pathways that are yet unknown [61]. The balance between the intrinsic downregulation of HLA molecules of the tumour cells and the effect of Interferon stimulation will eventually determine whether the circulating tumour cell is cleared by the immune system or not.

Fas also plays a role in immune evasion. Fas expression leads to recognition by, and activation of cytotoxic natural killer (NK) cells and promotes elimination from the circulation by the host immune system. Successful downregulation of the Fas molecule on the cell surface or corruption of downstream elements in the Fas pathway provides metastatic tumour cells with a survival advantage in the circulation and leads to an increase in metastatic potential. Patient samples from pulmonary OS metastases 
have been shown to be Fas-negative [40, 62]. Indeed, absence of Fas expression correlates with disease progression and poor survival outcome [23, 36, 55, 62].

\section{(Pre)clinical studies: Fas}

As the Fas receptor pathway is so important in the survival of metastatic cells, it is an attractive therapeutic target. Restoration of the Fas death pathway has been tried with success in preclinical models. Interleukin-12 (cytokine) therapy can achieve a dose-dependent upregulation of Fas on the surface of OS cells as well as a stimulation of cytotoxic T-cells and NK-cells. This renders the metastatic cells more sensitive to Fas-induced cell death in the microenvironment of the lung and enhances clearage of the cells from the circulation by the host immune system [36]. A drawback is the potent immunostimulatory effect of Interleukin-12 that can induce severe adverse effects after systemic administration in patients [56, 62].

In an in vivo experiment, intranasal administration of IL-12 resulted in Fas overexpression on OS lung metastases, leading to a decrease in tumour burden. Combination therapy with Ifosfamide, which induces the expression of FasL on the tumours, could further augment anti-tumour effect $[28,56]$.

IL-18 was reported to have similar effects on the activation of T-cells and NK-cells, as well as induction of the expression of FasL on already Fas expressing tumours. This compound did not, however, exert an anti-tumour effect in mice bearing OS lung metastases [63].

Gemcitabine is an agent that upregulates Fas-expression when administered as an aerosol therapy in mice bearing OS lung metastases. Gemcitabine aerosol therapy has been shown to effectively reduce size and number of pulmonary metastases [5, 55].

Liposomal MTP-PE (muramyl tripeptide phosphatidyl ethanolamine) is a promising agent for clinical use as it can induce endogenous IL-12 production and thus provide an up-regulation of Fas on OS cells but without the systemic toxicity encountered when exogenous IL-12 is administered to patients [28]. MTP-PE is a synthetic analogue of a component of bacterial cell walls. As an immunomodulatory agent it can also stimulate monocytes and macrophages to exert anti-tumour activity. The Children's Oncology Group performed a prospective randomised phase III clinical trial with this compound in patients with high-grade conventional OS with metastases at diagnosis. Treatment with liposomal MTP-PE improved overall survival, irrespective of the chemotherapy regimen. These data are promising and suggest that there is a critical role for the Fas death pathway in chemotherapy response which can be exploited in clinical practice to enhance the efficacy of chemotherapy in OS [40, 64, 65].

\section{(Pre)clinical studies: Immune modulation}

Modulation of the immune system to exert anti-tumour activity by the addition of interferon- $\alpha$ (IFN- $\alpha$ ) as a maintenance treatment after standard chemotherapeutic treatment is currently under investigation in the EURAMOS-1 trial, which is an initiative of the European and American Osteosarcoma Study Group [66]. IFN- $\alpha$ is immunomodulatory and able to stimulate a host-antitumour immune reaction and induce anti-proliferative signalling via the JAK/STAT1 pathway [58]. Accrual of patients for this worldwide trial is due to be completed in July $2011[61,66]$.

\section{(IV) Arrest and extravasation}

The mechanism of arrest of metastatic tumour cells at the distant organ sites remains controversial. One hypothesis is that metastatic cells are larger than ordinary cells in the circulation and that they become trapped in the microcirculation of a capillary bed. When trapped they form microembolisms and start interaction with the local environment. It is striking, however, that different tumour types have an organ specific preference for metastasis. The metastatic behaviour of OS is very distinct as over $80 \%$ of all metastases arise in the lungs and other organs usually remain unaffected. This suggests that the circulating tumour cell specifically 'homes' to distinct molecules that are expressed on the endothelium of the organ of preference. Although it might be trapped in different capillary beds throughout the body, it will interact with the surface molecules on the endothelium of the organ of interest rather than with endothelium at other sites [35]. There is evidence of endothelium-specific tropism in OS [10, 14, $15,22,42,67]$. The processes of exit of the circulation and invasion at the distant organ site are mediated by chemokines and proteinases. Proteinases are responsible for extravasation whereas chemokines determine the site at which circulating tumour cells adhere [9, 15]. Chemokines were initially thought to regulate leukocyte trafficking and homing, but recently they are also known as important components in the regulation of site-specific metastasis as they bind to G-protein coupled receptors on the plasma membrane of specific cells, in the case of OS to receptors in the lung [14, 42, 67-69]. CXCR-4, a commonly expressed chemokine in OS, is involved in site-specific metastasis. Its sole ligand is CXCL12 which is expressed abundantly in the lung. Binding of CXCR-4 to CXCL12 allows adherence and extravasation of OS cells in the lung [9, 10, 14, 15, 22, 42, 69]. Laverdiere et al. [42] found that CXCR-4 expression levels in patient samples inversely correlated to event-free and overall survival. There was a positive correlation between CXCR-4 expression in 
primary tumours and the presence of metastases at initial diagnosis. Interestingly, expression levels of CXCR-4 were similar in primary tumours and lung metastases. This suggests that CXCR-4 expression is not regulated during metastasis, but is simply present. It could be of predictive value for the formation of metastasis.

CXCR-3, another chemokine, is expressed by OS as well as other malignancies. Its ligands are CXCL9, -10 and -11 , all of which are expressed by lung, and this molecule is thought to co-operate with CXCR-4. Apart from mediating adherence, the interactions of CXCR-3 and -4 with their respective ligands also trigger pathways involved in other necessary events in metastasis, namely in invasion, survival and proliferation in the secondary tissue $[10,22,67]$.

For example, hypoxia upregulates CXCR-3 and -4 expression, which in turn induce the expression of MMP-2 and -9 on the cell surface and modulate the microenvironment into an inflammation-like condition, abundant with growth factors and stimulation of angiogenesis. Furthermore, binding of CXCR-4 to CXCL12 can activate the $\mathrm{NF}-\kappa \mathrm{B}$ survival pathway via ERK (Extracellular-signalRegulating-Kinase)-signalling and stimulate proliferation through MAPK signalling. Thus, apart from facilitating seeding at the distant organs site, chemokines play a very important role in the modulation of the microenvironment into a place permissive for the tumour cells to proliferate $[9$, $10,15,67]$.

\section{(Pre)clinical studies: Chemokines}

CXCR-4 is the most important chemokine-player in OS. Kim et al. [22] have demonstrated a reduction in metastatic tumour burden in an orthotopic mouse model in which cells were treated with a CXCR-4 inhibitor prior to injection of tumour cells into the mice. However, reduction of metastatic tumour burden without pre-treatment could not been shown consistently. The authors argue that the critical event, namely binding of CXCR-4 to CXCL12 with consecutive activation of signalling pathways, granting survival and proliferation, occurs too early in the establishment of metastases for inhibitory therapy of CXCR-4 to be beneficial for the patient with already existing metastasis. To what extent CXCR-4 inhibition could be beneficial in a preventive setting requires additional studies.

CXCR-3 inhibition was tested in an animal model for human OS lung metastases and showed a significant decrease in the development and progression of pulmonary lesions compared to the non-treated group [10].

\section{(V) Adherence}

Establishment at a distant organ requires the metastatic cell to connect to its new environment and re-establish cell-cell adhesions. Ezrin is a membrane-cytoskeleton linker protein that plays an important role in cell-microenvironment interaction. It is thought to facilitate anchorage of OS cells to lung tissue, as well as to enhance survival mechanisms in the new environment through Integrin mediated activation of Akt and MAPK survival pathways [14, 21, 25, 35]. The exact mechanism through which Ezrin mediates metastasis is not entirely clear, however, recently Wan et al. [70] discovered that $\beta 4$-Integrin is an important mediator. $\beta 4$-Integrin can bind Ezrin and Ezrin is required for the maintenance of this protein. $\beta 4$-Integrin can activate the PI3K pathway and thus stimulate survival and proliferation in the newly arrived cells in the lung. $\beta 4$-Integrin is found to be highly expressed in OS tumour samples from both primary and metastatic lesions. Furthermore, it was shown that $\beta 4$-Integrin knockdown inhibits the formation of OS lung metastasis in vivo, and leads to prolonged survival.

High expression of Ezrin correlated with a higher risk of metastatic relapse and poor survival in OS patients [21,25]. Furthermore, it was found to be 3 -fold overexpressed in lung metastases in a murine model for OS lung metastases [38]. CD44 is another surface molecule that can form a complex with Ezrin and correlates with metastasis and poor prognosis. Apart from influence on the cytoskeleton and cell shape, CD44 controls proliferation, growth arrest and survival via the Akt/mTOR pathway $[14,19,21]$.

\section{(Pre) clinical studies: Ezrin}

Suppression of Ezrin with a full-length anti-Ezrin construct did not inhibit primary tumour growth in a mouse model of OS, but it effectively inhibited the formation of metastases. It was speculated that metastatic OS cells express phosphorylated Ezrin only early after arrival in the lung, and this causes limited efficacy of suppression of Ezrin in readily established metastases, since its essential function in metastasis, namely connecting with the target organ site had already been fulfille [21]. Recently however, Ren et al. [25] suggested that Ezrin phosphorylation is not only present in the early stage of metastasis, but also late in tumour progression, at the leading edge of large metastasic lesions. This finding was verified on sections of patient OS metastases.

Pignochino et al. [45] reported that Sorafenib inhibited invasion via reduction in MMP-2 production and inhibited survival via downregulation of Ezrin-activated MAPK/Akt signalling. Furthermore, Sorafenib could also induce apoptosis in OS cells through downregulation of members of the anti-apoptotic Bcl-2 family. Wan et al. [70] showed that inhibition of Ezrin-related $\beta 4$-Integrin can reduce metastasis in a mouse model. Taken together, targeting Ezrin seems promising in the management of OS lung metastases. 


\section{(VI) Dormancy}

Dormancy refers to a prolonged period of survival of single cells or small micrometastases. OS patients can progress with metastases after a disease free interval of many years $[71,72]$. This is likely explained by the presence of micrometastases in a dormant state, which at some point are triggered develop into gross metastases.

Little is known concerning the biological processes regulating dormancy in OS. The anti-apoptotic gene Bcl-XL is thought to be involved in the survival of dormant cells, as well as $\alpha 5 \beta 1$-Integrin mediated activation of NF- $\kappa$ B. Furthermore the dormant state is thought to be regulated by the ratio between the ERK and p38-MAPK proteins, also steered by Integrin- $\alpha 5 \beta 1$ signalling [14, 35, 73, 74].

The mechanisms by which dormant tumour cells are at one point triggered to start proliferating are yet unaccounted for, however, the microenvironment is thought to play a regulatory role. Tumour outgrowth is dependent on vascularisation, and it has been suggested that endothelial cells in the microenvironment can both activate dormant tumour cells through cell-to-cell signalling and induce angiogenesis for nutrition [74]. The ECM is also thought to be involved in activation of dormant cells, as it serves as a source of growth and survival signals. It has been postulated that micrometastases that fail to properly connect to the ECM remain in the dormant state because they remain deprived of growth- and angiogenic signalling and go into quiescence as a means to survive. Anchorage to the ECM would stimulate cells to convert to a proliferative state via $\beta 1$-Integrin signalling [73]. The microenvironment can be regulated by the tumour cells themselves, but also by host stromal cells. Leucocytes and macrophages can modulate the ECM to either form a pro- or anti-angiogenic microenvironment. Apart from this, other mediating factors can also be influenced by stromal cells. For example, Wnt can be secreted from macrophages, and cytokines secreted by stromal cells can upregulate the intracellular $\mathrm{Wnt} / \beta$-catenin signalling pathway and hence induce survival and proliferation in a late stage in the process of metastasis $[9,35$, 54, 73]. Also, bone marrow derived progenitor cells (creating a 'pre-metastatic niche') can modulate the microenvironment and thus influence whether solitary cells or micrometastases remain dormant or are allowed to progress $[68,73]$. In an effort to elucidate the cellular mechanisms that establish the switch of dormant to rapidly growing cells, Almog et al. [75] designed an in vivo model for dormancy of various cancers, including OS, and performed gene-expression analysis of cells in the dormant state versus cells in a proliferative state. They found that during dormancy, there is an upregulation of anti-angiogenic proteins. In this pre-angiogenic situation, the tumour cells would lack the nutrition and oxygen needed to proliferate.
The cells that had switched to the proliferative phenotype had elevated RNA levels of common cancer pathways such as PI3K- and IGF-pathways. They also found that Endocan was upregulated in rapidly proliferating cells, a protein that is also expressed on tumour endothelial cells. This might indicate that endothelial changes support the switch of cells from dormancy into the proliferative state.

Dormancy could have a role in therapy resistance in OS metastases, however, whether this applies to OS and to what extent remains unknown. In general, dormancy can bring about drug resistance because non-proliferating cells are not so susceptible to conventional treatment. Most treatment modalities induce DNA damage which is usually more lethal to rapidly proliferating cells $[14,73,76]$. To intervene in this step of metastasis seems difficult. Angiogenesis seems to be an important factor. Elucidation of mechanisms that steer the switch from dormant to proliferative state may give some options. If it would be possible to keep the cells locked in the dormant state, it may grant the patient stable metastatic disease with prolonged survival.

\section{(VII) Angiogenesis and proliferation}

Tumour growth and progression is often restricted by vascularisation and thus nutrition. Hypoxia leads to the upregulation of growth factor receptors, angiogenic cytokines and proteolytic enzymes, among which EGFR, PDGF-R, VEGF, IGF-1R, TGF- $\beta$, IL-8 and MMPs, all of these providing neo-angiogenesis and allowing proliferation. These molecules can be overexpressed by the tumour cell population itself, but can also be provided by host endothelial (progenitor) cells during neo-angiogenesis [9, 14, 35, 40, 44, 68, 77]. Apart from induction of neoangiogenesis, VEGF also provides the tumour cells with a survival benefit via activation of ERK-1/2/NF- $\kappa \mathrm{B}$ and PI3K pathways [35].

Players involved in provision of vasculature and nourishment are often encountered in other processes within OS metastasis as well. For example, Src-kinase activity is regulated through various growth factor receptors, such as EGFR and Integrin receptors. Src activation leads to Ras/ MAPK signalling and activation of the transcription factor STAT3, allowing cell cycle progression and production of angiogenic factors such as fibroblast growth factor, VEGF and IL-8. Src phosphorylation by EGFR especially is considered to stimulate the onset of hyper-proliferation of tumour cells and induction of vascular permeability and neovascularisation [18, 44].

Proliferation of OS cells at a distant organ site is often mediated by Receptor-Tyrosine-Kinase or Integrin induced activation of PI3K and ERK1/2 pathways [9, 35, 67]. Alterations in cell cycle regulation can also promote proliferation by facilitating progression through the cell cycle 
checkpoints and speeding up the cycle. For example, the $\mathrm{Wnt} / \beta$-catenin pathway is of influence on both G1/S and G2/M progression via activation of Cyclin-D by c-myc and activation of Survivin respectively [46, 52].

The Insulin-Like-Growth-Factor 1 (IGF-1) Receptor axis is also implicated in the development of OS. It is striking that most OS arise during or shortly after puberty. The influence of GH and IGF-1 on bone growth steer the longitudinal growth during the adolescent growth spurt and contribute to approximately $50 \%$ of bone cell proliferation. As there is a peak incidence of OS during the adolescent growth spurt, it is conceivable that there could be GH/IGF-1 axis involvement in tumour development. IGF-1R signalling can activate the PI3K/Akt/mTOR pathway and stimulate survival and proliferation in tumour cells [40, 77-79].

\section{(Pre)clinical studies: Angiogenesis}

As OS is a highly vascularised tumour, a rationale exists to use this feature as a therapeutic target. High serum-VEGF levels correlate with metastatic relapse, tumour progression, poor response to chemotherapy and a decrease in survival $[40,77,80]$. Endostatin is an endogenous angiogenesis inhibitor, produced by tumours itself, involved in repression of neo-angiogenesis and is commonly expressed in human OS samples. It can also induce apoptosis in endothelial cells. Given its important role in angiogenesis, it was hypothesised that Endostatin could impair OS tumour growth and metastasis [18, 77, 81, 82]. However, in a murine model of OS lung metastasis, Endostatin failed to induce tumour shrinkage in the lungs, although, it did retard growth of lung nodules. Treatment with this drug will not cure OS patients, but it may result in stable metastatic disease with prolonged survival [83].

\section{(Pre)clinical studies: Proliferation}

Pharmacologic inhibition of the GH/IGF-1 axis and thus IGF-1R pathways has been explored. However, whereas there is evidence that IGF-1R signalling is important to primary OS growth, the extent to which IGF-1R (inhibition) could regulate OS metastases is not clear. In 2002, Mansky et al. [78] performed a phase I study in OS patients with metastatic and/or recurrent disease testing the clinical efficacy of the Somatostatin analog OncoLar. OncoLar was shown to significantly reduce circulating IGF-1 in patients. However, all patients enrolled showed disease progression.

More recently, fully humanised monoclonal antibodies (mABs) directed against the IGF-1R were tested in preclinical and clinical setting. In vivo IGF-1R inhibition with monoclonal antibodies induced growth retardation in subcutaneous models of OS [79, 84]. Whether this growth delay will also be shown in OS metastasis is unknown.
The SARC-011 clinical trial is evaluating the treatment effect of R1507, a mAB targeting the IGF-1R in patients with recurrent sarcomas, including OS (clinicaltrials.gov/ NCT00642941). In another clinical trial the efficacy of SCH717454, also targeting the IGF-1R, is evaluated in relapsed OS patients. In this trial, both inoperable patients and patients in whom metastasectomy is feasible are included. The latter group will be treated pre- and postmetastasectomy and might, apart from tumour response rate, give information about progression-free survival (clinicaltrials.gov/NCT00617890).

\section{Conclusion}

In conclusion, this review summarises potential molecular alterations that contribute to metastasis in OS and gives an overview of (pre)clinical efforts to develop new therapeutic targets for the treatment of metastatic OS. In spite of these efforts, OS metastasis is not yet well understood and there has been little evolvement in the treatment of this disease over the last decade. We hypothesise that certain molecular alterations seen in metastatic cells can also contribute to resistance to chemotherapy, and targeting these features might enhance the efficacy of current treatments. Further unravelling the biology of OS metastasis will hopefully provide new insights to be used as a rational basis for innovative metastasis directed treatments for OS.

Acknowledgements We would like to acknowledge prof. E.S Kleinerman (M.D. Anderson Cancer Center, Houston, TX, USA) for her input and guidance in the preparation of this manuscript. JP is supported by the Individualised Musculoskeletal Regeneration and Reconstruction Network (Danish Research Council) Aarhus, Denmark and by VONK: VUmc Onderzoek naar Kinderkanker (Stichting Research Fonds Kindergeneeskunde VUmc) Amsterdam, the Netherlands.

Open Access This article is distributed under the terms of the Creative Commons Attribution Noncommercial License which permits any noncommercial use, distribution, and reproduction in any medium, provided the original author(s) and source are credited.

\section{References}

1. Mirabello L, Troisi RJ, Savage SA (2009) Osteosarcoma incidence and survival rates from 1973 to 2004: data from the Surveillance, Epidemiology, and End Results Program. Cancer 115(7):1531-1543

2. Bacci G, Rocca M, Salone M et al (2008) High grade osteosarcoma of the extremities with lung metastases at presentation: treatment with neoadjuvant chemotherapy and simultaneous resection of primary and metastatic lesions. J Surg Oncol 98(6):415-420

3. Bielack SS, Carrle D, Hardes J et al (2008) Bone tumors in adolescents and young adults. Curr Treat Options Oncol 9(1):67-80 
4. Harting MT, Blakely ML (2006) Management of osteosarcoma pulmonary metastases. Semin Pediatr Surg 15(1):25-29

5. Hughes DP (2009) Strategies for the targeted delivery of therapeutics for osteosarcoma. Expert Opin Drug Deliv 6(12):13111321

6. Messerschmitt PJ, Garcia RM, bdul-Karim FW et al (2009) Osteosarcoma. J Am Acad Orthop Surg 17(8):515-527

7. Kager L, Zoubek A, Potschger U et al (2003) Primary metastatic osteosarcoma: presentation and outcome of patients treated on neoadjuvant Cooperative Osteosarcoma Study Group protocols. J Clin Oncol 21(10):2011-2018

8. Bielack SS, Kempf-Bielack B, Delling G et al (2002) Prognostic factors in high-grade osteosarcoma of the extremities or trunk: an analysis of 1,702 patients treated on neoadjuvant cooperative osteosarcoma study group protocols. J Clin Oncol 20(3):776-790

9. Eccles SA, Welch DR (2007) Metastasis: recent discoveries and novel treatment strategies. Lancet 369(9574):1742-1757

10. Pradelli E, Karimdjee-Soilihi B, Michiels JF et al (2009) Antagonism of chemokine receptor CXCR3 inhibits osteosarcoma metastasis to lungs. Int J Cancer 125(11):2586-2594

11. Rodriguez NI, Hoots WK, Koshkina NV et al (2008) COX-2 expression correlates with survival in patients with osteosarcoma lung metastases. J Pediatr Hematol Oncol 30(7):507-512

12. Fuchs J, Seitz G, Ellerkamp V et al (2008) Analysis of sternotomy as treatment option for the resection of bilateral pulmonary metastases in pediatric solid tumors. Surg Oncol 17(4):323-330

13. Briccoli A, Rocca M, Salone M et al (2010) High grade osteosarcoma of the extremities metastatic to the lung: long-term results in 323 patients treated combining surgery and chemotherapy, 1985-2005. Surg Oncol 19(4):193-199

14. Krishnan K, Khanna C, Helman LJ (2005) The biology of metastases in pediatric sarcomas. Cancer J 11(4):306-313

15. deNigris F, Rossiello R, Schiano C et al (2008) Deletion of Yin Yang 1 protein in osteosarcoma cells on cell invasion and CXCR4/angiogenesis and metastasis. Cancer Res 68(6):17971808

16. Diaz-Montero CM, Wygant JN, McIntyre BW (2006) PI3-K/Aktmediated anoikis resistance of human osteosarcoma cells requires Src activation. Eur J Cancer 42(10):1491-1500

17. Guo Y, Rubin EM, Xie J et al (2008) Dominant negative LRP5 decreases tumorigenicity and metastasis of osteosarcoma in an animal model. Clin Orthop Relat Res 466(9):2039-2045

18. Hingorani P, Zhang W, Gorlick R et al (2009) Inhibition of Src phosphorylation alters metastatic potential of osteosarcoma in vitro but not in vivo. Clin Cancer Res 15(10):3416-3422

19. Kansara M, Thomas DM (2007) Molecular pathogenesis of osteosarcoma. DNA Cell Biol 26(1):1-18

20. Kansara M, Tsang M, Kodjabachian L et al (2009) Wnt inhibitory factor 1 is epigenetically silenced in human osteosarcoma, and targeted disruption accelerates osteosarcomagenesis in mice. J Clin Invest 119(4):837-851

21. Khanna C, Wan X, Bose S et al (2004) The membrane-cytoskeleton linker ezrin is necessary for osteosarcoma metastasis. Nat Med 10(2):182-186

22. Kim SY, Lee CH, Midura BV et al (2008) Inhibition of the CXCR4/CXCL12 chemokine pathway reduces the development of murine pulmonary metastases. Clin Exp Metastasis 25(3):201-211

23. Lafleur EA, Koshkina NV, Stewart J et al (2004) Increased Fas expression reduces the metastatic potential of human osteosarcoma cells. Clin Cancer Res 10(23):8114-8119

24. Pasello M, Michelacci F, Scionti I et al (2008) Overcoming glutathione S-transferase P1-related cisplatin resistance in osteosarcoma. Cancer Res 68(16):6661-6668

25. Ren L, Hong SH, Cassavaugh J et al (2009) The actin-cytoskeleton linker protein ezrin is regulated during osteosarcoma metastasis by PKC. Oncogene 28(6):792-802
26. Wang IC, Chen YJ, Hughes DE et al (2008) FoxM1 regulates transcription of JNK1 to promote the G1/S transition and tumor cell invasiveness. J Biol Chem 283(30):20770-20778

27. Zucchini C, Rocchi A, Manara MC et al (2008) Apoptotic genes as potential markers of metastatic phenotype in human osteosarcoma cell lines. Int J Oncol 32(1):17-31

28. Gorlick R, Anderson P, Andrulis I et al (2003) Biology of childhood osteogenic sarcoma and potential targets for therapeutic development: meeting summary. Clin Cancer Res 9(15):5442-5453

29. Kawabe T (2004) G2 checkpoint abrogators as anticancer drugs. Mol Cancer Ther 3(4):513-519

30. Nyberg KA, Michelson RJ, Putnam CW et al (2002) Toward maintaining the genome: DNA damage and replication checkpoints. Annu Rev Genet 36:617-656

31. Reinhardt HC, Aslanian AS, Lees JA et al (2007) p53-deficient cells rely on ATM- and ATR-mediated checkpoint signaling through the p38MAPK/MK2 pathway for survival after DNA damage. Cancer Cell 11(2):175-189

32. Syljuasen RG, Jensen S, Bartek J et al (2006) Adaptation to the ionizing radiation-induced G2 checkpoint occurs in human cells and depends on checkpoint kinase 1 and Polo-like kinase 1 kinases. Cancer Res 66(21):10253-10257

33. Hanahan D, Weinberg RA (2000) The hallmarks of cancer. Cell 100(1):57-70

34. Kashima T, Nakamura K, Kawaguchi J et al (2003) Overexpression of cadherins suppresses pulmonary metastasis of osteosarcoma in vivo. Int J Cancer 104(2):147-154

35. Steeg PS (2006) Tumor metastasis: mechanistic insights and clinical challenges. Nat Med 12(8):895-904

36. Worth LL, Lafleur EA, Jia SF et al (2002) Fas expression inversely correlates with metastatic potential in osteosarcoma cells. Oncol Rep 9(4):823-827

37. Hynes RO (2003) Metastatic potential: generic predisposition of the primary tumor or rare, metastatic variants-or both? Cell 113(7):821-823

38. Khanna C, Khan J, Nguyen P et al (2001) Metastasis-associated differences in gene expression in a murine model of osteosarcoma. Cancer Res 61(9):3750-3759

39. Minn AJ, Gupta GP, Siegel PM et al (2005) Genes that mediate breast cancer metastasis to lung. Nature 436(7050):518-524

40. Anderson P, Kopp L, Anderson N et al (2008) Novel bone cancer drugs: investigational agents and control paradigms for primary bone sarcomas (Ewing's sarcoma and osteosarcoma). Expert Opin Investig Drugs 17(11):1703-1715

41. Hughes DP (2010) How the NOTCH pathway contributes to the ability of osteosarcoma cells to metastasize. Cancer Treat Res 152:479-496

42. Laverdiere C, Hoang BH, Yang R et al (2005) Messenger RNA expression levels of CXCR4 correlate with metastatic behavior and outcome in patients with osteosarcoma. Clin Cancer Res 11(7):2561-2567

43. Fan DG, Dai JY, Tang J et al (2009) Silencing of calpain expression reduces the metastatic potential of human osteosarcoma cells. Cell Biol Int 33(12):1263-1267

44. Kim LC, Song L, Haura EB (2009) Src kinases as therapeutic targets for cancer. Nat Rev Clin Oncol 6(10):587-595

45. Pignochino Y, Grignani G, Cavalloni G et al (2009) Sorafenib blocks tumour growth, angiogenesis and metastatic potential in preclinical models of osteosarcoma through a mechanism potentially involving the inhibition of ERK1/2, MCL-1 and ezrin pathways. Mol Cancer 8:118

46. Rubin EM, Guo Y, Tu K et al (2010) Wnt inhibitory factor 1 decreases tumorigenesis and metastasis in osteosarcoma. Mol Cancer Ther 9(3):731-741

47. Guo Y, Zi X, Koontz Z et al (2007) Blocking Wnt/LRP5 signaling by a soluble receptor modulates the epithelial to 
mesenchymal transition and suppresses met and metalloproteinases in osteosarcoma Saos-2 cells. J Orthop Res 25(7):964-971

48. Engin F, Bertin T, Ma O et al (2009) Notch signaling contributes to the pathogenesis of human osteosarcomas. Hum Mol Genet 18(8):1464-1470

49. Tanaka M, Setoguchi T, Hirotsu M et al (2009) Inhibition of Notch pathway prevents osteosarcoma growth by cell cycle regulation. Br J Cancer 100(12):1957-1965

50. Zhang P, Yang Y, Zweidler-McKay PA et al (2008) Critical role of notch signaling in osteosarcoma invasion and metastasis. Clin Cancer Res 14(10):2962-2969

51. Igney FH, Krammer PH (2002) Death and anti-death: tumour resistance to apoptosis. Nat Rev Cancer 2(4):277-288

52. Leow PC, Tian Q, Ong ZY, Yang Z, Ee PL (2010) Antitumor activity of natural compounds, curcumin and PKF118-310, as Wnt/ $\beta$-catenin antagonists against human osteosarcoma cells. Invest New Drugs 28(6):766-782

53. Cai Y, Mohseny AB, Karperien M et al (2010) Inactive Wnt/betacatenin pathway in conventional high-grade osteosarcoma. J Pathol 220(1):24-33

54. Jeanes A, Gottardi CJ, Yap AS (2008) Cadherins and cancer: how does cadherin dysfunction promote tumor progression? Oncogene 27(55):6920-6929

55. Gordon N, Koshkina NV, Jia SF et al (2007) Corruption of the Fas pathway delays the pulmonary clearance of murine osteosarcoma cells, enhances their metastatic potential, and reduces the effect of aerosol gemcitabine. Clin Cancer Res 13(15 Pt 1):4503-4510

56. Duan X, Jia SF, Koshkina $N$ et al (2006) Intranasal interleukin-12 gene therapy enhanced the activity of ifosfamide against osteosarcoma lung metastases. Cancer 106(6):1382-1388

57. Yang C, Yang S, Wood KB et al (2009) Multidrug resistant osteosarcoma cell lines exhibit deficiency of GADD45alpha expression. Apoptosis 14(1):124-133

58. Yuan XW, Zhu XF, Huang XF et al (2007) Interferon-alpha enhances sensitivity of human osteosarcoma U2OS cells to doxorubicin by p53-dependent apoptosis. Acta Pharmacol Sin 28(11):1835-1841

59. Hoang BH, Kubo T, Healey JH et al (2004) Expression of LDL receptor-related protein 5 (LRP5) as a novel marker for disease progression in high-grade osteosarcoma. Int $\mathrm{J}$ Cancer 109(1):106-111

60. Schuetze S, Wathen K, Choy E et al (2010) Results of a Sarcoma Alliance for Research through Collaboration (SARC) phase II trial of dasatinib in previously treated, high-grade, advanced sarcoma. J Clin Oncol 28(15):10009

61. Whelan J, Patterson D, Perisoglou M et al (2010) The role of interferons in the treatment of osteosarcoma. Pediatr Blood Cancer 54(3):350-354

62. Lafleur EA, Jia SF, Worth LL et al (2001) Interleukin (IL)-12 and IL-12 gene transfer up-regulate Fas expression in human osteosarcoma and breast cancer cells. Cancer Res 61(10):4066-4071

63. Nakamura Y, Yamada N, Ohyama H et al (2006) Effect of interleukin-18 on metastasis of mouse osteosarcoma cells. Cancer Immunol Immunother 55(9):1151-1158

64. Chou AJ, Kleinerman ES, Krailo MD et al (2009) Addition of muramyl tripeptide to chemotherapy for patients with newly diagnosed metastatic osteosarcoma: a report from the Children's Oncology Group. Cancer 115(22):5339-5348

65. Lewis VO (2009) What's new in musculoskeletal oncology. J Bone Joint Surg Am 91(6):1546-1556
66. EURAMOS. www.ctu.mrc.ac.uk/euramos. Accessed 18 May 2009. Ref Type: Electronic Citation

67. Huang CY, Lee CY, Chen MY et al (2009) Stromal cell-derived factor-1/CXCR4 enhanced motility of human osteosarcoma cells involves MEK1/2, ERK and NF-kappaB-dependent pathways. J Cell Physiol 221(1):204-212

68. Kaplan RN, Psaila B, Lyden D (2006) Bone marrow cells in the 'pre-metastatic niche': within bone and beyond. Cancer Metastasis Rev 25(4):521-529

69. Murphy PM (2001) Chemokines and the molecular basis of cancer metastasis. N Engl J Med 345(11):833-835

70. Wan X, Kim SY, Guenther LM et al (2009) Beta4 integrin promotes osteosarcoma metastasis and interacts with ezrin. Oncogene 28(38):3401-3411

71. Hauben EI, Bielack S, Grimer R et al (2006) Clinico-histologic parameters of osteosarcoma patients with late relapse. Eur J Cancer 42(4):460-466

72. Strauss SJ, McTiernan A, Whelan JS (2004) Late relapse of osteosarcoma: implications for follow-up and screening. Pediatr Blood Cancer 43(6):692-697

73. Barkan D, Green JE, Chambers AF (2010) Extracellular matrix: a gatekeeper in the transition from dormancy to metastatic growth. Eur J Cancer 46(7):1181-1188

74. Favaro E, Amadori A, Indraccolo S (2008) Cellular interactions in the vascular niche: implications in the regulation of tumor dormancy. APMIS 116(7-8):648-659

75. Almog N, Ma L, Raychowdhury R et al (2009) Transcriptional switch of dormant tumors to fast-growing angiogenic phenotype. Cancer Res 69(3):836-844

76. Liu X, Lei M, Erikson RL (2006) Normal cells, but not cancer cells, survive severe Plk1 depletion. Mol Cell Biol 26(6):20932108

77. Scotlandi K, Picci P, Kovar H (2009) Targeted therapies in bone sarcomas. Curr Cancer Drug Targets 9(7):843-853

78. Mansky PJ, Liewehr DJ, Steinberg SM et al (2002) Treatment of metastatic osteosarcoma with the somatostatin analog OncoLar: significant reduction of insulin-like growth factor-1 serum levels. J Pediatr Hematol Oncol 24(6):440-446

79. Kolb EA, Gorlick R, Houghton PJ et al (2008) Initial testing (stage 1) of a monoclonal antibody ( $\mathrm{SCH} 717454$ ) against the IGF-1 receptor by the pediatric preclinical testing program. Pediatr Blood Cancer 50(6):1190-1197

80. Abdeen A, Chou AJ, Healey JH et al (2009) Correlation between clinical outcome and growth factor pathway expression in osteogenic sarcoma. Cancer 115(22):5243-5250

81. Kim HS, Lim SJ, Park YK (2009) Anti-angiogenic factor endostatin in osteosarcoma. APMIS 117(10):716-723

82. Ryu K, Choy E, Yang C, Susa M, Hornicek FJ, Mankin H, Duan Z (2010) Activation of signal transducer and activator of transcription 3 (Stat3) pathway in osteosarcoma cells and overexpression of phosphorylated-Stat 3 correlates with poor prognosis. J Orthop Res 28(7):971-978

83. Kaya M, Wada T, Nagoya S et al (2007) Prevention of postoperative progression of pulmonary metastases in osteosarcoma by antiangiogenic therapy using endostatin. J Orthop Sci 12(6):562567

84. Kolb EA, Kamara D, Zhang W et al (2010) R1507, a fully human monoclonal antibody targeting IGF-1R, is effective alone and in combination with rapamycin in inhibiting growth of osteosarcoma xenografts. Pediatr Blood Cancer 55(1):67-75 